\title{
Dihadron production in semi-inclusive DIS from transversely polarized protons
}

\author{
S. Gliske ${ }^{* a b}$ and L. Pappalardo ${ }^{c}$ (on behalf of the HERMES Collaboration) \\ a High Energy Physics Division, \\ Argonne National Laboratory \\ Lemont, Illinois, USA \\ ${ }^{b}$ University of Michigan \\ Ann Arbor, Michigan, USA \\ ${ }^{c}$ Dipartimento di Fisica e Scienze della Terra \\ Università di Ferrara \\ 44122 Ferrara, Italy \\ E-mail: sgliske@umich.edy, pappalardo@fe.infn.it
}

\begin{abstract}
Transverse Momentum Dependent (TMD) dihadron production, including vector meson production, allows access to various TMD distribution and fragmentation functions. Dihadron production is complementary to single hadron semi-inclusive DIS measurements, pairing the same distribution functions with different fragmentation functions. While dihadrons present unique measurement opportunities, the TMD dihadron cross section is significantly more complex than that for single hadron production, due to the polarization in the final state. Various theoretical advances, which further clarify the complexity, will be highlighted. The HERMES analysis of the transverse target moments of the TMD dihadron cross section allows the first test of a particular prediction of the Lund/Artru string fragmentation model, specifically that the favored Collins fragmentation function has opposite sign in single hadron production versus vector meson production. The status and results of this analysis will be discussed and an extension of the Lund/Artru model for disfavored fragmentation will also be presented.
\end{abstract}

XXI International Workshop on Deep-Inelastic Scattering and Related Subject-DIS2013, 22-26 April 2013

Marseilles,France

\footnotetext{
* Speaker.
} 


\section{Introduction}

Semi-inclusive deep-inelastic scattering (SIDIS) accesses combinations of distribution functions and fragmentation functions [1]. SIDIS dihadron production, constituting both resonant and non-resonant hadron pairs, is not only complimentary to SIDIS pseudo-scalar production but also provides unique opportunities. For example, measurements from both the dihadron and pseudoscalar transverse momentum dependent (TMD) cross section are needed to test the Lund/Artru string fragmentation model [2]. Additionally, dihadron production allows collinear access to transversity, which is not possible with single pseudo-scalar production. Collinear-based global fits are advantageous as the evolution equations are not known in the TMD case.

\section{Fragmentation Models}

The Lund/Artru string fragmentation model posits that, as a transversely polarized struck quark is exiting the proton, a gluon flux tube breaks into a quark, anti-quark pair with quantum numbers equal to that of the vacuum, $0^{++}$. Angular momentum conservation implies that if the produced anti-quark has spin parallel to the struck quark (i.e. a $\left|\frac{1}{2}\right\rangle\left|\frac{1}{2}\right\rangle$ or $\left|\frac{1}{2}\right\rangle\left|\frac{1}{2}\right\rangle$ state), the produced hadron will prefer moving towards the left, while if the spins are anti-parallel (i.e. a $\left|\frac{1}{2}\right\rangle\left|-\frac{1}{2}\right\rangle$ or $\left|-\frac{1}{2}\right\rangle\left|\frac{1}{2}\right\rangle$ state), the produced hadron will move towards the right. Relating the direct sum basis with the direct product basis results in the conclusion that pseudo-scalar mesons $\left(|0,0\rangle \propto\left|\frac{1}{2}\right\rangle\left|-\frac{1}{2}\right\rangle+\left|-\frac{1}{2}\right\rangle\left|\frac{1}{2}\right\rangle\right)$ will prefer moving towards the right while the two transversely polarized vector meson states $\left(|1, \pm 1\rangle=\left| \pm \frac{1}{2}\right\rangle\left| \pm \frac{1}{2}\right\rangle\right)$ will prefer moving towards the left, implying the Collins function has opposite sign for these two cases.

Note however, that the Lund/Artru model assumes that the struck quark is in the produced hadron, typical for favored fragmentation but not for disfavored fragmentation. A model for disfavored fragmentation, denoted the Gliske Gluon Radiation model, can be obtained by assuming the hadron is produced from the quark, anti-quark pair produced from the vacuum [3], i.e. the struck quark emitting a high energy, off-shell gluon and the quark returns to the proton remnant. Further soft-interactions which do not effect the spin set additional quantum numbers. A common sub-diagram is shared between the Lund/Artru model and this gluon radiation model. Applying the same angular momentum and spin states arguments to this model results in the prediction for the Collins function sign as does the Lund/Artru model. Although these models are mainly focused on first rank fragmentation, one can assume higher rank fragmentation to be mostly negligible in determining the sign of the Collins function. Thus, the combination of these two models suggests that the Collins function for both favored and disfavored transverse $(|1, \pm 1\rangle)$ vector meson states have the same sign, opposite to the sign of the Collins function for favored single pseudo-scalar hadron production [卂].

\section{Theory}

The Lund/Artru and Gliske Gluon Radiation string fragmentation models are both amplitude level models, not cross section level which involves the amplitude times its complex conjugate. To relate the models with the cross section, a modified definition of the distribution functions is introduced [3, 5], where the quark, quark-conjugate spins define the fragmentation function labels and 
all final-state polarization is subsumed in the partial wave expansions. This new convention merits a new partial wave analysis including the full $\ell$ and $m$ specification of the final-state polarization [3, 5].

In particular, the collinear interference fragmentation function $H_{1}^{\Varangle}$ [6] is associated with the $|\ell=1, m=1\rangle$ partial wave of the Collins function, while the $|2, \pm 2\rangle$ partial waves of the Collins function are those which are expected to have opposite sign as the pseudo-scalar Collins function. The new partial wave expansion also highlights the existing fact that the $H_{1}^{\Varangle}$, i.e. $H_{1}^{|1,1\rangle}$, receives contributions from both $s p$ and $p p$ interference.

This new partial wave analysis also capitalizes on the inherent symmetries in the SIDIS cross section. Specifically, the cross section without any partial wave expansion has identical form to the cross section for producing a pure scalar final state, i.e. single pseudo-scalar production. Thus, one can compute the cross section for any final-state polarization, at any twist, by 1) obtaining the cross section for single pseudo-scalar production at the desired twist-level, 2) setting the non-expanded, polarized final-state cross section to have identical form, and 3) expanding the fragmentation functions according to spherical harmonics.

Using this method, the dihadron cross section, including twist-2 and twist-3, for all final-state polarizations has been computed. The transverse-target moments were published in Ref. [5], the unpolarized moments were discussed in the presentation corresponding to these proceedings, and the publication of the complete expressions is in progress. The collinear $|1,1\rangle$ moment related to the Collins function and transversity coincide with the $\sin \left(\phi_{R}+\phi_{S}\right) \sin \vartheta$ modulation, while the TMD $|2, \pm 2\rangle$ moments coincide with the $\sin \left((1 \mp 2) \phi_{h} \pm 2 \phi_{R}+\phi_{S}\right) \sin ^{2} \vartheta$ modulations. The angle definitions are as in Ref. [5].

The remainder of this document focuses on the analysis of $\pi^{ \pm} \pi^{0}$ and $\pi^{+} \pi^{-}$dihadrons, subsuming the $\rho$-triplet resonances. Note, the convention is that for a $h_{1} h_{2}$ di-meson dihadron, assuming $h_{1}$ and $h_{2}$ do not have equal charge, $h_{1}$ refers to the charged meson when one of the mesons is neutral, and $h_{1}$ refers to the positively charged meson when both $h_{1}$ and $h_{2}$ are charged.

\section{Analysis}

Data was taken with the HERMES detector using the HERA $27.6 \mathrm{GeV}$ lepton beam during the years 2002-2005 with a transversely polarized hydrogen target. Lepton-hadron separation efficiency was better than $98 \%$, and hadron identification was made using an event-level algorithm and the ring-imaging Cerenkov (RICH) detector. Events were required to pass data quality and SIDIS identification cuts $\left(Q^{2}>1 \mathrm{GeV}^{2}, W^{2}>10 \mathrm{GeV}^{2}, 0.023<x<0.4,0.2<y<0.95,0.2<z<0.8\right.$, $\left.0.05 \mathrm{GeV}<P_{h \perp}<1.60 \mathrm{GeV}\right)$. The $\pi^{0}$-mesons were identified as a resonant peak in the diphoton mass spectrum, and the $\pi^{ \pm} \pi^{0}$ data were corrected for non-resonant diphoton pairs. The charge symmetric background and exclusive background were determined to be negligible.

The effect of the HERMES acceptance on the angular variables was corrected in the parameter space of the amplitudes rather than in the histogrammed yield space of Ref. [团. Both approaches are mathematically equivalent, but the parameter-space approach is desirable in higher dimensions - dihadrons analysis involves four angular variables and currently uses 42 angular moments in the fit function (though more moments are present in the cross section), while pseudoscalar hadron analysis only involves one or two angular variables (depending on the target polar- 


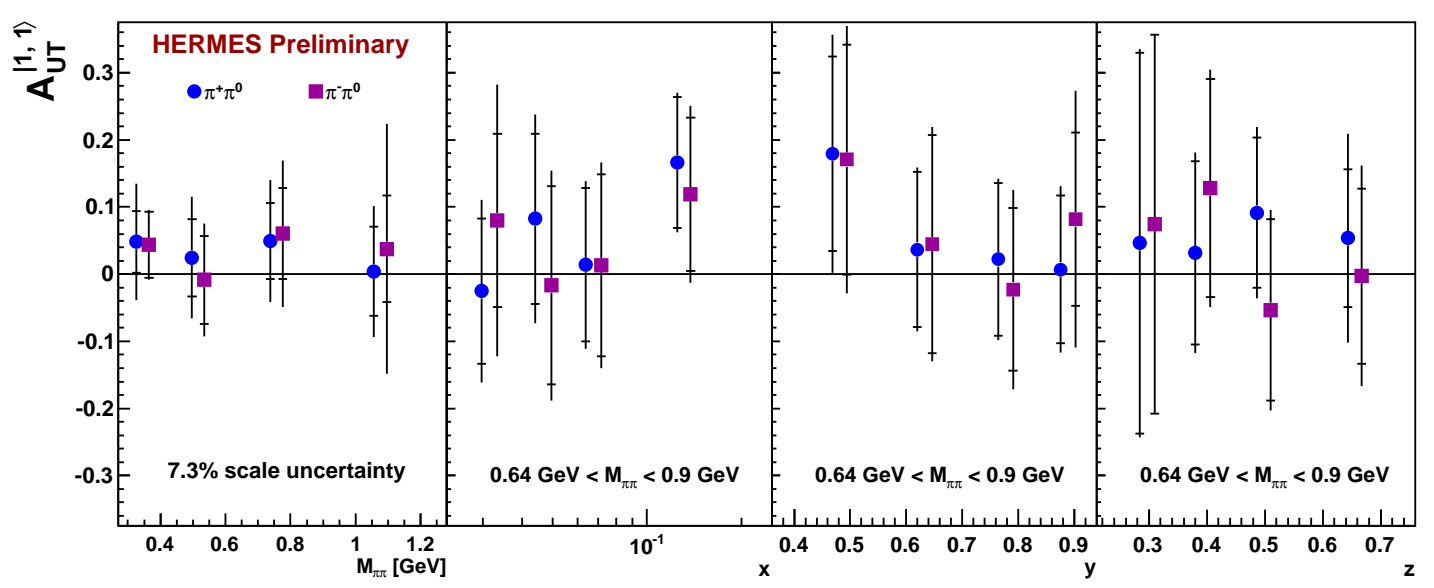

Figure 1: The $\sin \left(\phi_{R}+\phi_{S}\right) \sin \vartheta$ amplitudes for $\pi^{ \pm} \pi^{0}$ dihadrons. The left panel shows the results versus the invariant mass of the $\pi \pi$ system, $M_{\pi \pi}$, while the right three panels show the $x, y$, and $z$ dependence of the data restricted to the mass bin centered at the $\rho$-mass. Full error bars are the statistical uncertainty (inner error bar) added in quadrature with the systematic uncertainty.

ization). The 42 moments used in this analysis are the 24 twist- 2 and twist- 3 unpolarized moments and the 18 (twist-2) Collins and Sivers transverse target moments.

The acceptance correction required the development of a new Monte Carlo generator, TMDGen, as well as a new TMD spectator model for dihadron fragmentation. Both TMDGen and the spectator model are detailed in Refs. [3, 5]. Smearing and radiative effects are considered in the systematic uncertainty, but no corrections for those effects were applied. Systematic uncertainties also consider the variation in results between the running period with positron instead of electron beams, as well as uncertainties related to the RICH hadron identification.

\section{Results}

The results for $\pi^{ \pm} \pi^{0}$ dihadron $|1,1\rangle$ amplitude related to transversity and the Collins function are shown in Figure 1. The $\pi^{+} \pi^{-}$results are not shown, as they were previously published [8] and the new analysis uses a more optimized choice of binning. The signs of the amplitudes are consistent across all three $\pi \pi$ dihadrons, although the statistics are more limited for the $\pi^{ \pm} \pi^{0}$ dihadrons than for the $\pi^{+} \pi^{-}$dihadrons. These results are suitable to be included in global, collinear extractions of transversity and may further constrain the flavor separation.

The results for $|2, \pm 2\rangle$ Collins amplitudes are shown in Figure 2. The results are consistent with zero outside of the $\rho$-mass peak for both $|2, \pm 2\rangle$ amplitudes, suggesting that the non-resonant background has no observable presence in this partial wave. The $|2,-2\rangle$ amplitude is also consistent with zero in the $\rho$-mass peak. One explanation is that the transversity distribution function causes the struck quark to be in the up-state, causing the $|2,-2\rangle$ state to be inaccessible and thus zero. The $|2,2\rangle$ amplitude in the $\rho$-mass peak for $\pi^{ \pm} \pi^{0}$ dihadrons are indicate a non-zero, negative value. The results are indeed consistent with the expectations of the Lund/Artru and Gluon Radiation models and encourage repeating these measurements at existing or future facilities. 


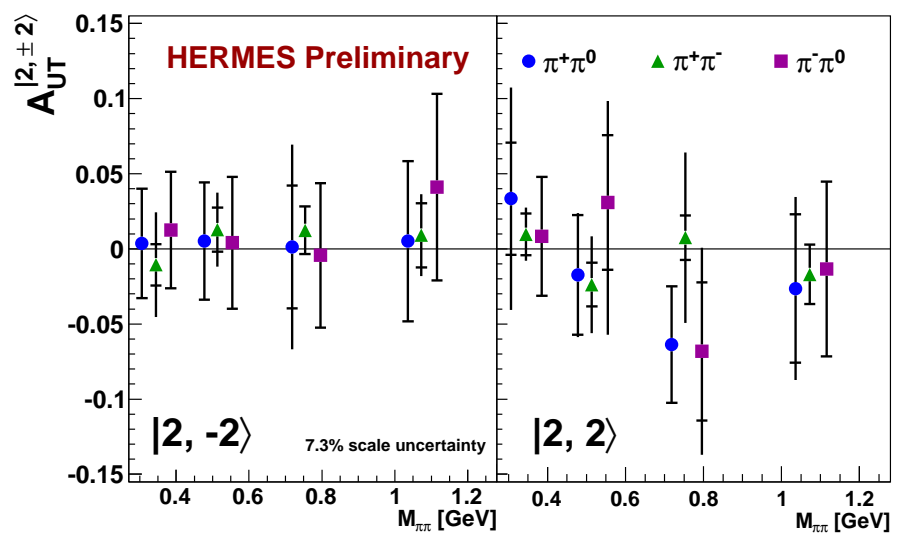

Figure 2: The $\sin \left(3 \phi_{h}-2 \phi_{R}+\phi_{S}\right) \sin ^{2} \vartheta$ (left panel) and $\sin \left(-\phi_{h}+2 \phi_{R}+\phi_{S}\right) \sin ^{2} \vartheta$ (right panel) amplitudes for $\pi \pi$ dihadrons versus the invariant mass of the $\pi \pi$ system. Error bars are plotted as in Figure 1.

\section{Acknowledgments}

We gratefully acknowledge the DESY management for its support, the staff at DESY and the collaborating institutions for their significant effort, and our national funding agencies and the EU FP7 (HadronPhysics2, Grant Agreement number 227431) for financial support.

\section{References}

[1] A. Bacchetta et al., Semi-inclusive deep inelastic scattering at small transverse momentum, JHEP 093 (2007) [arXiv: hep-ph/0611265].

[2] X. Artru, Proposals for measuring transversity distributions in deep inelastic electron scattering and a model for E-704 asymmetries, in proceedings of $V^{\text {th }}$ Intl. Workshop on High Energy Spin Physics (SPIN-93), Protvino, Russia (1993) [arXiv: hep-ph / 9310323 ].

[3] S. Gliske, Transverse Target Moments of Dihadron Production in Semi-inclusive Deep Inelastic Scattering at HERMES, PhD dissertation, University of Michigan (2011) http://deepblue.lib.umich.edu/handle/2027.42/86394.

[4] A. Airapetian et al. (Hermes Collaboration), Effects of transversity in deep-inelastic scattering by polarized protons, PLB 693 (2010) 11 [arXiv: 1006.4221 ].

[5] S. Gliske for the HERMES Collaboration, Transverse Target Moments of SIDIS Vector Meson Production at HERMES, in proceedings of The $19^{\text {th }}$ International Spin Physics Symposium (SPIN 2010), JoP: Conf. Series. 295 (2011) 012044 http://stacks.iop.org/1742-6596/295/i=1/a=012044.

[6] A. Bacchetta and M. Radici, Modeling dihadron fragmentation functions, Phys. Rev. D 74 (2006) 114007 [arXiv: hep-ph/0608037].

[7] A. Airapetian et al. (Hermes Collaboration), Azimuthal distributions of charged hadrons, pions, and kaons produced in deep-inelastic scattering off unpolarized protons and deuterons, Phys. Rev. D 87 (2013) 012010 [arXiv:1204.4161].

[8] A. Airapetian et al. (HeRmes Collaboration), Evidence for a transverse single-spin asymmetry in leptoproduction of $\pi^{+} \pi^{-}$pairs, JHEP 06 (2008) 017 [arXiv: 0803.2367 ]. 